\title{
Perceptions of Rural Emergency Department Providers regarding Telehealth-Based Care: Perceived Competency, Satisfaction with Care and Tele-ED Patient Disposition
}

\author{
Roseanne Fairchild1, Shiaw Fen Ferng Kuo², Stephanie Laws³, Amanda O'Brien³, \\ Hicham Rahmouni ${ }^{3}$ \\ ${ }^{1}$ Nursing and Health Professions, State College of Florida, Manatee-Sarasota, Sarasota, FL, USA \\ ${ }^{2}$ Department of Applied Health Sciences, Indiana State University, Terre Haute, IN, USA \\ ${ }^{3}$ Union Hospital, Richard Lugar Center for Rural Health, Terre Haute, IN, USA \\ Email: fairchr@scf.edu,s-ferng@indstate.edu
}

How to cite this paper: Fairchild, R., Kuo, S.F.F., Laws, S., O'Brien, A. and Rahmouni, H. (2017) Perceptions of Rural Emergency Department Providers regarding Telehealth-Based Care: Perceived Competency, Satisfaction with Care and Tele-ED Patient Disposition. Open Journal of Nursing, 7, 721-733.

https://doi.org/10.4236/ojn.2017.77054

Received: May 3, 2017

Accepted: July 4, 2017

Published: July 7, 2017

Copyright $\odot 2017$ by authors and Scientific Research Publishing Inc. This work is licensed under the Creative Commons Attribution International License (CC BY 4.0).

http://creativecommons.org/licenses/by/4.0/

\begin{abstract}
Introduction: This study assesses rural providers' perceptions of their ability to deliver high quality care via telehealth compared to usual care, and whether attending providers perceive that emergency department (ED) telehealth visits influence clinical reasoning in regard to patient disposition, specifically in tele-behavioral and tele-neurological cases. Methods: A cross-sectional survey was conducted of $134 \mathrm{ED}$ providers (nurses $[n=126]$ and physicians $[n=8]$ ) who were working in five Midwestern critical access hospitals (response rate $83 \%)$. Descriptive, correlational and stepwise regression analyses were employed to evaluate provider perceptions of 1) competency level in telehealth delivery, 2) patient health outcomes, 3) access to continuing education in telehealth, and 4) clinical influence of telehealth visit. Evaluation of preliminary set of $N=100$ telehealth cases were assessed for influence of telehealth on clinical reasoning of attending physicians regarding patient disposition. Results: The majority $(67 \% ; n=90)$ of participants had at least minimal experience with telehealth care delivery, with an average of $1-2$ visits in teleneurology, and $3-4$ visits in telebehavioral cases. Providers rated their overall mean competency level in telehealth care delivery as $3.01 / 5.00$ based on a 5 point "novice (1) to expert" (5) scale. Mean scores for providers perceived competency level in 7 evidence-based sub-categories for telehealth care delivery were self-reported as relatively low to mid-range values, ranging from $2.64-3.57 / 5.00$. Stepwise linear regression analysis of whether all providers "would recommend telehealth to their family and friends" revealed two predictors for model of best fit $\left(n=81 ; p<0.000 ; \mathrm{R}^{2}=0.598\right)$ : 1$)$ their percep-
\end{abstract}


tions of telehealth experience compared to usual care; and 2) perceptions of patient health outcomes with telehealth compared to usual care. Providers rated "neutral" to "very unlikely" that they "would recommend telehealth to family and friends" $(2.75 / 5.00 ; n=122 ; 91 \%)$. Attending physicians reported that for a majority of cases, telehealth visits influenced patient disposition and transfer decision-making (58.4\%), and the influence of telehealth visits on patient disposition was statistically significantly higher for behavioral health cases $(p<0.018)$. Discussion: This study will be followed on to inform administrators/policy makers about 1) perceived level of competency of providers who implement tele-emergency care, 2) potential importance of telehealth equipment used and teamwork between rural providers and distant specialist, and 3) how use of telehealth may enhance ability of rural ED providers to improve quality of care. Perceived influence of telehealth on patient disposition is reported to be highest for telebehavioral patients. Healthcare educators need to place a priority on addressing provider competencies in telehealth through health professions degree programs and continuing education. Further research is needed to promote application and testing of evidence-based provider competencies in telehealth, and potentially relevant health communication models, to increase providers' perceived efficacy and competency in telehealth care delivery, thus supporting high quality patient health outcomes.

\section{Keywords}

Telehealth, Telemedicine, Rural Emergency Department, Critical Access

Hospital, Provider Competency, Attending Physician, Patient

Disposition, Telebehavioral, Teleneurological

\section{Introduction}

The use and applications of telehealth services have expanded over the past twenty years, along with the role of technology in improving access to, and delivering healthcare. Telehealth has improved a healthcare organization's capacity to increase access to specialty care, reduce or eliminate patient's travel time to tertiary facilities, and improve quality of care through targeted provider support. [1] The traditional model of telehealth is of primary consideration for the current study, and involved care delivered "virtually" via a secure, online internet-based audio/video connection to emergency department patients at a series of originating sites, from appropriately licensed specialists working at distant sites.

Public and private payers currently reimburse providers for certain services delivered through telehealth, and the shift from a fee-for-service system to accountable quality of care systems in the United States has the potential to increase utilization of telehealth. [1] In the U.S., reimbursement programs discouraging hospital readmissions and high numbers of preventable emergency department (ED) visits are examples of how shifting incentives away from providing a high volume of services can encourage use of telehealth to improve patient 
outcomes, while potentially reducing costs. [1] Although telehealth services utilization has grown, there remains a need for robust, replicable telehealth studies in rural and/or medically underserved areas that can inform provider preparation and care delivery, especially as the emphasis on quality of care and cost effectiveness continues to increase [1] [2].

\subsection{Current Applications of Telehealth: Emergency Departments}

Ekeland and colleagues [2] recently concluded that telemedicine (telehealth) has been demonstrated to be effective in behavioral health counseling and management of chronic diseases. However, there is a need for more evidence regarding the effectiveness of telehealth to support delivery of emergent care in behavioral health (e.g., suicide attempt, overdose), neurology (e.g., stroke, seizure), and trauma (injury) care in rural emergency departments (EDs). [2] A recent analysis of telehealth applications in the U.S. showed that $32 \%$ of the 4727 reporting hospitals are using at least one type of telehealth service, and services are currently being utilized in approximately $8 \%$ of emergency departments. [3] While utilization of telehealth is on the rise, a systematic review of telemedicine studies revealed that "high-quality evidence to inform policy decisions on how best to use [telehealth/telemedicine] in health care is still lacking". [2] In addition, although most hospitals participating in telehealth-based programs are in rural settings, [4] [5] many studies reporting on telehealth-based programs often focus on larger urban healthcare systems due to larger patient volumes, and thus, enhanced capacities for statistical analysis [5].

One recent U.S. rural hospital survey wherein 63,193 ED cases were examined for frequency counts revealed that the most common diagnoses treated using telehealth were mental health, trauma, circulatory issues and presentation of complex signs or symptoms ( $n=1512$ telehealth cases). [6] This descriptive assessment involved 21 rural critical access hospitals, and also highlighted thematic interview data that a sample of rural providers $(n=85)$ believed that telehealth helped support rapid patient transfers. [6] No quantitative analysis was included regarding patient disposition decision-making, perceptions of telehealth-based outcomes compared to usual care, nor perceptions of provider-level competencies in telehealth care delivery in the clinical setting.

\subsection{Telehealth and Provider Perceptions of Competency of Care}

While tele-emergent care has been identified as an ideal use of telehealth for rapid consultation with care specialists, there is a need for more in-depth evaluation of the major implications regarding implementation and outcomes of the utilization of tele-emergency care. [6] Medicare has also suggested tele-emergency care as ideal for quick consultation with rural sites; [5] however, provider perceptions of their competency level in regard to their continuing education needs in telehealth, and the potential influence of the telehealth visit on different types of patient cases, have not been thoroughly investigated, including perceptions of the ability to deliver high quality care via telehealth 
compared to usual care [1] [2] [3].

\subsection{Purpose of the Study}

The purpose of this study was to inform rural emergency care providers, administrators and policy makers about the perceived competency level of providers who implement emergency care via telehealth for behavioral health and neurological patients, as well as the attending providers' perceptions of the influence of the telehealth visit on clinical reasoning in relation to patient disposition and patient health outcomes. As part of a multi-phase, longitudinal study, these efforts should allow healthcare leaders and policy makers to begin to more strategically support evidence-based national and regional policies and procedures in healthcare provider telehealth education and practice that may positively influence the ability of rural providers to improve the quality and efficiency of care that patients receive in U.S. critical access hospitals (CAHs).

\section{Methods}

This cross-sectional study examined perceptions of educational preparation in telehealth and telehealth care delivery for ED providers working in five Midwest U.S. CAH EDs during October-December, 2015, to determine baseline perceptions of: 1) Competency level in utilization of telehealth applications; 2) educational preparation and access to continuing education in telehealth; 3) number of clinical experiences with telehealth; 4) patient health outcomes compared to usual care; 5) the telehealth visit's influence on the attending provider (physician) in regard to their clinical decision-making related to patient disposition for the two primary types of cases (telebehavioral and teleneurological); 6) and request for healthcare provider comments to help reveal additional information surrounding the overall context for the optimal delivery of telehealth-based care. Ethical institutional review board approval for the study was obtained prior to the onset of study.

\subsection{Study Participants}

Emergency department provider role characteristics are presented in Figure 1 for those participating in the study in five Midwestern CAH EDs. All providers were engaged in the majority of their work time in the rural EDs, and were comprised of nurses $(n=109)$, physicians $(n=8)$ and other ancillary ED providers, such as respiratory therapy and nursing assistants. Participants were $84 \%$ female $(n=112)$ and $16 \%$ male $(n=22)$. Participation in the study was voluntary, and all interested ED personnel completed an informational webinar describing the purpose of the study, and those volunteering to participate provided written informed consent prior to the launch of study activities.

\subsection{Equipment}

A traditional model of telehealth care delivery was utilized for the current study, and involved care delivered "virtually" via secure, online internet-based Tandberg/ 


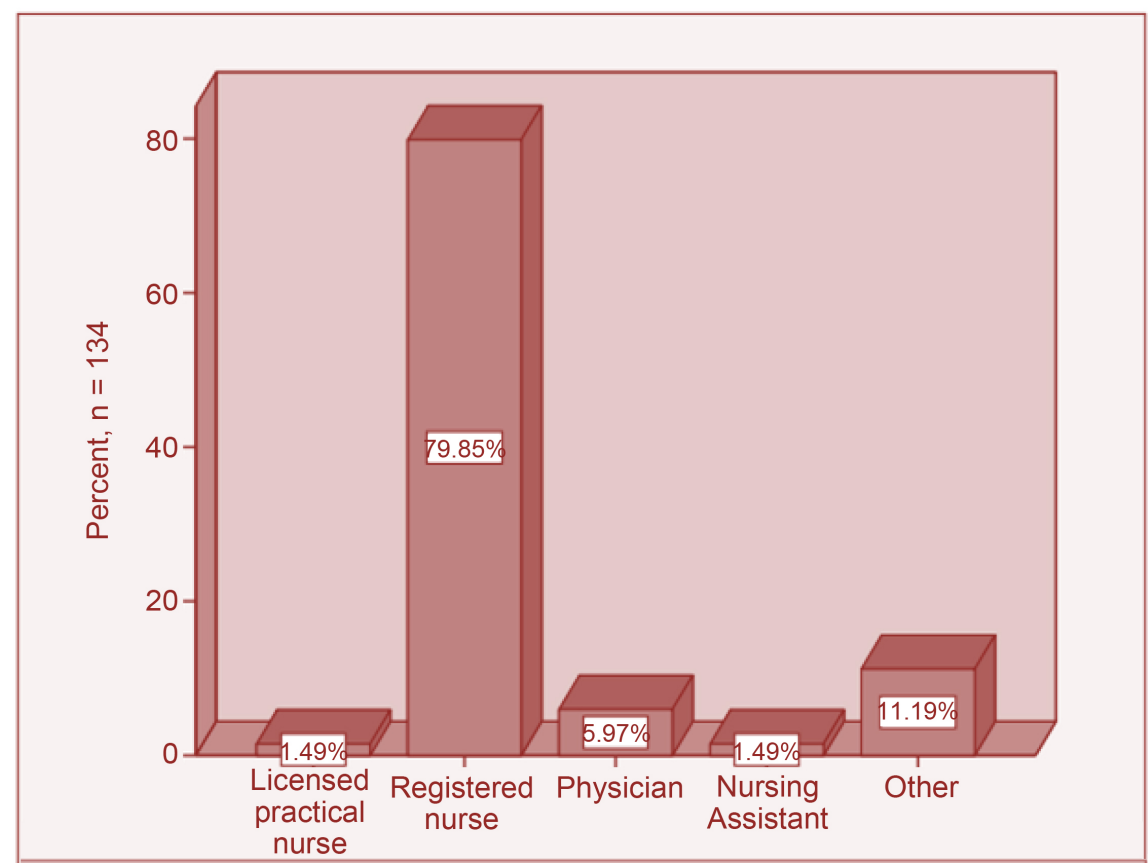

Figure 1. Professional role distribution of participants.

Cisco videoconferencing infrastructure, sometimes called "computers on wheels" (COWs), with bandwidth ranging from $364 \mathrm{Kbs}-720 \mathrm{~Kb} / \mathrm{s}$ utilizing point-topoint fiber optic connections and Ethernet service to connect the patient and health care team at each rural hospital's originating site to an appropriately licensed specialist working at a distant site. Rather than a "store and forward" approach to telehealth care delivery, all participating rural hospitals utilized "live video interaction" in "real time" to activate a telehealth visit with the desired specialist for the patient, as determined by the attending provider (rural ED physician).

\subsection{Provider Survey}

A 30-item, 5-point Likert-type password-protected online survey, the "Inventory for Tele-Emergent Care (ITEC)" [7], which had been refined by the authors in a series of CAH pilot studies during 2012-2014, was implemented during Fall, 2015, to evaluate rural ED provider perceptions of: 1) competency level in telebehavioral and teleneurological applications (5 point scale, "novice" to "expert" in 7 sub-categories); 2) presence of telehealth education in their health professions degree program (5 point scale, "strongly agree" to "strongly disagree"; 3 ) access to continuing education in telehealth from one or more sources ( 5 point scale, "strongly agree" to "strongly disagree"); 4) degree of clinical experience with the 2 different telehealth care categories ( 5 point scale, " 0 visits" to " $12+$ visits" performed); and 5) patient health outcomes utilizing telehealth were comparable to usual care (5 point scale, "strongly agree" to "strongly disagree"). Internal consistency reliability was 0.93 for the ITEC survey instrument. 
Attending physicians were also asked to rate the following probability statements on a 5-point Likert-type scale for each of the initial telehealth patient cases $(n=100)$ treated during the first 6 months of the study: 1$)$ Prior to the telehealth visit, their intention was to transfer the patient; and 2) How much the telehealth visit influenced their clinical decision-making in relation to patient disposition. An open-ended question asking participants if there was any additional information they wanted to share was also included at the end of the provider survey.

Of a total of 162 providers polled who completed written informed consent to voluntarily participate in the survey and the telehealth case evaluations, 144 accessed and 134 completed survey, including registered nurses $(n=107)$, physicians $(n=8)$ and other ED providers $(n=19)$ engaged in telehealth delivery, demonstrating a response rate of $83 \%$. With a $4 \%$ margin of error, the 134 valid samples reflects statistical power greater than $90 \%$.

\subsection{Data Analysis}

The Statistical Package for the Social Sciences (SPSS) version 22.0 was utilized to evaluate for differences, similarities and statistically significant relationships between and among the study variables. Interrelationships explored included the ED provider's educational preparation in telehealth care delivery and access to continuing education in telehealth, perceptions of telehealth-based care compared to usual care, and the potential influence of the telehealth visit on the attending provider's decision-making based on type of case being treated in the ED. Null hypotheses were stated for all variable relationships before analyses were conducted, with statistical significance set at $p<0.05$. Any additional information or comments that participants shared at the end of the quantitative survey were also analyzed for content and major themes, to help provide context for the quantitative survey results.

\section{Results}

\subsection{Educational Preparation}

A majority of participants had not participated in continuing education (CE) in telehealth during the past year $(n=81 ; 62.3 \%)$, nor was their educational preparation likely to have included telehealth practice experiences $(n=88 ; 69.3 \%)$. Providers' reported that their comfort level with telehealth care delivery was associated with engagement in continuing education (CE) $(p<0.004)$. Overall, providers' self-reported experience with telehealth care delivery varied widely, and ranged from 0 visits performed to more than 12 visits performed based on survey results.

\subsection{Provider Perceptions of Telehealth Care Delivery}

The majority $(67 \% ; n=90)$ of participants had at least minimal experience with telehealth care delivery, with an average of 1 - 2 visits in teleneurology, and 3 - 4 visits in telebehavioral cases. Providers rated their overall mean competency lev- 
el in telehealth care delivery as $3.01 / 5.00$ based on a 5 point "novice (1) to expert" (5) scale. In addition, mean scores for providers perceived competency level in 7 evidence-based sub-categories [1] [2] for telehealth care delivery, including communications (patient-provider and provider-provider), understanding use of the equipment/technology, critical thinking skills needed, clinical judgment skills needed, quality of care delivery, and privacy/confidentiality, were self-reported as relatively low to mid-range values, ranging from $2.64-3.57 / 5.00$ (Table 1).

In regard to the potential predictors for providers' perceived tendency to recommend telehealth-based care, stepwise regression analysis revealed the following: For telebehavioral patients, ED providers' perceptions of 1) their level of

Table 1. Summary of providers' perceived competency for telehealth care delivery.

\begin{tabular}{|c|c|c|c|c|c|c|c|c|c|}
\hline \multirow[b]{2}{*}{ Items } & \multirow[b]{2}{*}{ Provider Competencies } & \multicolumn{5}{|c|}{$\begin{array}{l}\text { Likert Scale Items } \\
\text { (\% Respondents) }\end{array}$} & \multicolumn{3}{|c|}{ Statistics } \\
\hline & & 1 & 2 & 3 & 4 & 5 & Mean & $\begin{array}{c}\alpha \\
(\text { t test } 1)^{*}\end{array}$ & $\begin{array}{c}\alpha \\
(t \text { test } 2)^{* *}\end{array}$ \\
\hline 1 & $\begin{array}{l}\text { Communication skills } \\
\text { with patient during the } \\
\text { telehealth visit }\end{array}$ & 22.1 & 11.5 & 39.7 & 22.1 & 4.6 & $2.76 \pm 1.16$ & $0.003^{*}$ & $0.000^{* *}$ \\
\hline 2 & $\begin{array}{l}\text { Communication skills } \\
\text { with other provider(s) } \\
\text { during the } \\
\text { telehealth visit }\end{array}$ & 20.6 & 12.2 & 37.4 & 25.2 & 4.6 & $2.81 \pm 1.16$ & $0.016^{*}$ & $0.000^{* *}$ \\
\hline 3 & $\begin{array}{l}\text { Understanding any } \\
\text { limitations of the } \\
\text { equipment/technology } \\
\text { in order to make a } \\
\text { sound care decision }\end{array}$ & 22.9 & 16.8 & 38.2 & 17.6 & 4.6 & $2.64 \pm 1.15$ & $0.001^{*}$ & $0.000^{* *}$ \\
\hline 4 & Critical thinking skills & 6.9 & 4.6 & 38.2 & 38.2 & 12.2 & $3.44 \pm 1.00$ & $0.008^{*}$ & $0.010^{* *}$ \\
\hline 5 & $\begin{array}{l}\text { Clinical judgment } \\
\text { regarding decisions } \\
\text { about when a } \\
\text { patient may require an } \\
\text { in-person evaluation }\end{array}$ & 6.9 & 4.6 & 44.6 & 36.9 & 6.9 & $3.32 \pm 0.93$ & $0.015^{*}$ & $0.002^{* *}$ \\
\hline 6 & $\begin{array}{l}\text { Adhering to the same } \\
\text { quality of care standards } \\
\text { as for an in-person visit }\end{array}$ & 5.3 & 5.3 & 48.9 & 34.4 & 6.1 & $3.31 \pm 0.88$ & 0.127 & $0.007^{\star *}$ \\
\hline 7 & $\begin{array}{l}\text { Adhering to the same } \\
\text { standards of patient } \\
\text { privacy and } \\
\text { confidentiality }\end{array}$ & 4.6 & 4.6 & 38.5 & 33.8 & 18.5 & $3.57 \pm 1.00$ & 0.418 & 0.123 \\
\hline
\end{tabular}

${ }^{*} t$ test 1: Significant difference between providers answering "Yes" for Q: "Did your educational preparation address competencies for quality of telehealth care delivery in any of your degree programs?" and providers answering "No". Statistical. significance level is $0.05 .{ }^{* *}$ t test 2 : Significant difference between providers answering "Yes" for Q: "Have you had any continuing education to address competencies for quality of telehealth care delivery in any facilities where you have worked?" and providers answering "No". Statistical significance level is 0.05 . 
experience in telehealth care delivery, and 2) patient health outcomes compared to usual care, were statistically significant predictors for the statement "would recommend telehealth to family and friends" ( $\left.n=81 ; p<0.001 ; \mathrm{R}^{2}=0.598\right)$. For teleneurological patients, ED providers' level of experience in telehealth care delivery experiences was the only predictor $\left(n=39 ; p<0.001 ; \mathrm{R}^{2}=0.642\right)$ for the statement "would recommend telehealth to family and friends". Overall, from a descriptive standpoint, providers rated "neutral" to "very unlikely" that they "would recommend telehealth to family and friends" ( $\mu=2.75 / 5.00 ; n=122$; 91\%).

In additional stepwise regression analysis for care of telebehavioral patients, ED providers' perceptions of their 1) competency level in telehealth and 2) following the evidence-based protocol for the telehealth visit, were significant predictors for "overall comfort level with telehealth care delivery" $(n=81 ; p<$ $\left.0.001 ; R^{2}=0.598\right)$. For teleneurological patients, ED providers' perceptions of their level of experience in telehealth care delivery was a statistically significant predictor $\left(n=39 ; p<0.001 ; \mathrm{R}^{2}=0.642\right)$ for their "overall comfort level with telehealth care delivery".

\subsection{Influence of Telehealth Visit: Attending Provider Decision-Making}

Attending physicians were also asked to rate the probability that the telehealth visit influenced their clinical decision-making in relation to patient disposition for this initial phase of the study ( $N=100$ patient cases; $n=61$ telebehavioral, $n=$ 39 teleneurological). Attending physicians reported that for these cases, a majority of the telehealth visits influenced patient disposition and transfer decisionmaking $(58 \% ; n=58)$. In addition, it is important to note that the reported influence of telehealth visits on patient disposition was statistically significantly higher for behavioral health cases $(p<0.018)$. The most common diagnoses for which a telehealth visit was activated by the rural ED attending provider were 1) Behavioral health diagnoses: overdose, either accidental or intentional; suicidal ideation or suicide attempt; substance abuse; depression; anxiety; and 2) Neurological diagnoses: stroke (CVA) and transient ischemic attack (TIA).

\subsection{Contextual Comments by Participating Providers}

A content analysis of the free-form comments provided by the participating providers at the end of the survey was performed to determine major themes surrounding providers' perceptions of telehealth-based care. Three major themes were revealed by the providers, which supplied important context for future modifications of the mode of telehealth care delivery for this longitudinal study. Example narratives supporting the themes are provided below.

\subsubsection{Technical/Equipment Issues}

Several of the healthcare provider participants complained about the cumbersome nature of, and the time and effort involved in, utilizing and manipulating all the technical features needed on the "COWs" to deliver the telehealth visits. 
In addition, numerous audio and/or video internet connection issues were described, to such an extent that providers would become discouraged at times and were reluctant to actually attempt to utilize and conduct a telehealth-based visit, because they felt it sometimes negatively impacted patient care. One nurse expressed the following frustrations: "It needs to work well. You can not [sic] expect to meet pt needs using telemedicine if it doesn't work right most of the time. Telebehavioral works best but teleneurology rarely works and unlike telehealth I think neurology consults should be done in person.” Another provider shared that "Telebehavior needs to be quicker-after we fax our information there is still a wait time of several hours for the pt-many pt's [sic] make comments about not being important enough or get angry enough they demand to leavethis opens up all kinds of problems-having to call security or the police etc. The end result is that the pt [sic] is feeling worse than when they came in. And admission could have happened hours before."

\subsubsection{Lack of Responsiveness/Collegiality: Distantsite Specialist(s)}

Several providers also reported that specialists may not initially respond to phone calls when a telehealth visit was initiated, and that when the specialist did finally call in, 30 minutes were typically required for a call-back to deliver the care, especially if the visit was requested during the night shift. Moreover, when some of the specialists called in, the providers perceived that the specialist was annoyed and/or was not behaving in a collegial manner with the distant rural team, especially when they had questions and/or requested more information from the specialist on call. Some providers stated that, "it's a lack of availability of teleneuro, long delays in response or sometimes no response at all", and "it's the timeliness of consults. Sometimes it takes a while for a consult to begin, they do not respond very quickly. When we have technical issues, this does not set well and they [specialists] get upset".

\subsubsection{Need for Continuing Education/Technical Education}

Several providers also asked for and/or expressed a need for continuing education in regard to implementation of telehealth-based visits in regard to best practices, and also in regard to being able to use the equipment and the various features available on the "COWs" telemedicine cart. A physician and a nurse commented, respectively, "We need sufficient education for staff to increase comfort level for usage and advantages of tele-health", and "Too much time is spent trying to identify and troubleshoot problems with the equipment which can be detrimental to the patient."

\section{Discussion}

In this study, a majority of ED providers self-reported an average to low level of comfort with telehealth care delivery, and the majority are unlikely to recommend telehealth to family and friends. However, based on the contextual comments by several providers, ED healthcare staff may be more likely to recommend and successfully implement telehealth-based care with equipment that is 
easier to utilize, with improved and upgraded audio/video connectivity capabilities, and/or when self-reported need for continuing education is provided to support best practices in technical applications of the equipment, and, ultimately, evidence-based implementation of telehealth care delivery.

\subsection{Equipment and Technical Difficulties}

While participants in one large project in tele-emergent care implementation reported few technical issues in telehealth-based care delivery, [8] the author reported surveying only hospital administrators rather than the ED staff who are responsible for actually using telehealth equipment and implementing visits (end-users). A more recent study revealed that technical difficulties in telehealth care delivery can result from inadequate bandwidth for the internet connection, [9] thus underscoring the potential need to bolster this aspect of the rural hospital-based and community-based infrastructures supporting the technology when implementing a telehealth program.

While Ward and colleagues [10] recently reported positive findings for a systematic review of telehealth studies relating to technical quality and end-user satisfaction, some U.S. rural hospitals may not have the financial resources to keep up with technological improvements in equipment and/or internet platforms currently available for seamless implementation of telehealth care delivery. Based on these initial outcomes and provider concerns in relation to technical issues for delivery of telehealth-based care in the participating rural hospitals, alternatives to the current COW set-ups, and current internet platform capabilities that are being utilized in the rural hospital EDs, will be considered by the Midwest rural telehealth network and research team.

\subsection{Continuing Education for Healthcare Providers}

With the advent of technologically-driven healthcare, continuing education in telehealth care delivery methodologies and equipment for providers is not only practical, it is a professional imperative. [8] [9] [11] Duchesne and colleagues [9] described a significant learning curve in regard to the utilization of telehealthbased equipment, and observed that in addition, the all-too-familiar calls for excellence in teamwork and communication between rural providers and the distant site specialist were essential to the success of their rural telemedicine program in the U.S. state of Mississippi.

Henry and colleagues [11] recently systematically evaluated a set of 45 studies worldwide relating to healthcare provider behaviors, perceptions and concerns in regard to telehealth care delivery from a qualitative perspective. While the authors believed they could not yet present a set of best practices for clinician behaviors in regard to telehealth care delivery, they emphasized the following emerging themes as important considerations to improving provider education, as well as the quality of care, to support optimal utilization of telehealth, including: "Perceptions of the utility of telehealth; differences in communication patterns such as pace and type of discourse; reliance on visual cues by both provider 
and patient especially in communicating empathy and building rapport; and confidentiality and privacy in telehealth care delivery" [11].

While an emphasis seems to have been placed on continuing education for telehealth team end-users to promote excellence in telehealth care delivery, further research is needed to determine an evidence-based set of best practices to support healthcare professionals' initial exposure to, and continuing education in, the implementation of telehealth-based care. Moreover, some experts suggest that beyond technical training and education to promote development of best practices, and to maximize the impact of end-user uptake of telehealth, digitized modes of care such as telehealth must actually be integrated into the culture of an organization [12].

The study team therefore recommends the consideration and testing of different health behavior communication models with telehealth provider teams in particular. Further research in this realm would help providers move beyond simply implementing basic algorithms of care in telehealth for various health conditions, to testing relevant health behavior communication models to promote high quality, virtual telehealth-based care for patients and families. This approach should help inter professional healthcare teams collectively implement and evaluate an evidence-based communications model as a best practice in telehealth for providers and distant site specialists, promoting excellence in patient health outcomes, as well as in professional telehealth practice in a variety of healthcare settings. As Wade and colleagues' [13] recent qualitative study reminds us, "clinician acceptance" is key to fostering sustainability in telehealth care delivery.

\subsection{Limitations of the Study}

This study was limited to participation of a majority of ED providers in five U.S. critical access hospitals, and as such the results are not necessarily generalizable to ED providers working in all U.S. critical access hospitals. Further study is needed to help discern the important educational needs of rural and urban providers who deliver patient care via telehealth. In addition, the ITEC survey tool is available from the researchers and needs further testing to bolster support for its validity and reliability in regard to the 7 sub-categories of factors tentatively proposed to influence provider perceptions of telehealth care delivery. However, the authors believe that these initial results may help lay the foundation for future research in regard to development of tools that are useful in telehealth program evaluation in regard to providers' educational needs, as well as provider perceptions of different types of telehealth care delivery compared to usual (face-to-face) care.

\subsection{Conclusion}

Continuing education in telehealth is an educational imperative, with the current emphasis on health information technologies to enhance efforts to improve patient health outcomes in rural CAH EDs. While technical difficulties may be 
addressed with improvements in telehealth platforms and equipment in future planning with rural CAHs, important work also lies ahead in regard to enhancing rural site-distant site teamwork in rural settings, promoting engagement in telehealth-based continuing education, and in testing and adopting health communication models for astute telehealth practice. Communication (verbal and nonverbal) is key to successful telehealth care delivery, as it underscores and drives cultural and behavioral health understanding in professional care delivery settings. Working on our collective communication from a practical standpoint represents an entrée into the organization's ability to achieve an optimal level of cultural integration of telehealth care delivery.

\section{Acknowledgements}

The authors wish to thank the healthcare administrators and staff of the participating critical access hospital emergency departments, as well as technical support personnel, for their dedication to their patients and contributions to the research.

\section{Declaration of Conflicting Interests}

The author(s) declared no potential conflicts of interest with respect to the research, authorship, and/or publication of this article.

\section{Funding}

This study was supported by the Health Resource and Services Administration (HRSA) of the U.S. Department of Health and Human Services (HHS) under grant number G01RH27871 and title Evidence-Based Tele-emergency Network Grant Program. The content and conclusions of this study are those of the authors and should not be construed as the official position or policy of, not should any endorsement be inferred by HRSA, HHS, or the U.S. Government.

\section{References}

[1] Broderick, A. and Lindeman, D. (2013) Scaling Tele-Health Programs: Lessons from Early Adopters. The Commonwealth Fund, Publication 1654, Vol. 1.

[2] Ekeland, A.G., Bowes, A. and Flottorp, S. (2010) Effectiveness of Telemedicine: A Systematic Review of Reviews. International Journal of Medical Informatics, 79, 736-771.

[3] Mueller, K.J., Potter, A.J., MacKinney, A.C. and Ward, M.M. (2014) Lessons from Tele-Emergency: Improving Care Quality and Health Outcomes by Expanding Support for Rural Care Systems. Health Affairs, 33, 228-234. https://doi.org/10.1377/hlthaff.2013.1016

[4] Easton, J.D., Saver, J.L., Alvers, G.W., Alberts, M.J., Chaturvedi, S., Fledmann, E., Hatsukami, T.S., Higashida, R.T., Johnston, S.C., Kidwell, C.S., Lutsep, H.L., Miller, E. and Sacco, R.L. (2009) Definition and Evaluation of Transient Ischemic Attack: A Scientific Statement for Healthcare Professionals from the American Heart Association/American Stroke Association Stroke Council. Stroke, 40, 2276-2293.

https://doi.org/10.1161/STROKEAHA.108.192218 
[5] Gliman, M. and Stensland, J. (2013) Telehealth and Medicare: Payment Policy, Current Use, and Prospects for Growth. MMRR, 3, E1-E17. https://doi.org/10.5600/mmrr.003.04.a04

[6] Ward, M.M., Ullrich, F., MacKinney, A.C., Bell, A.L., Shipp, S. and Mueller, K.J. (2016) Tele-Emergency Utilization: In What Clinical Situations Is Tele-Emergency Activated? Journal of Telemedicine and Telecare, 22, 25-31. https://doi.org/10.1177/1357633X15586319

[7] Fairchild, R., Ferng, S.F. and Laws, S. (2012-2014) Pilot Testing and Factor Analysis for the Inventory for Tele-Emergent Care in Critical Access Hospitals. Unpublished Pilot Study Data.

[8] Henderson, K. (2006) TelEmergency: Distance Emergency Care in Rural Emergency Departments Using Nurse Practitioners. Journal of Emergency Nursing, 32, 388393.

[9] Duchesne, J.C., Kyle, A., Simmons, J., Islam, S., Schmieg, R.E., Olivier, J. and McSwain, N.E. (2008) Impact of Telemedicine upon Rural Trauma Care. The Journal of Trauma, 64, 92-97.

[10] Ward, M.M., Jaana, M. and Natafgi, N. (2015) Systematic Review of Telemedicine Applications in Emergency Rooms. International Journal of Medical Informatics, 84, 601-616.

[11] Henry, B.W., Block, D.E., Ciesla, J.R., McGowan, B.A. and Vozenilek, J.A. (2016) Clinician Behaviors in Telehealth Care Delivery: A Systematic Review. Advances in Health Sciences Education, 1-20. https://doi.org/10.1007/s10459-016-9717-2

[12] Newman, L., Bigardadi, N. and Schraeder, G. (2016) Service Providers' Experiences Using a Telehealth Network 12 Months after Digitization of a Large Australian Rural Mental Health Service. International Journal of Medical Informatics, 94, 8-20.

[13] Wade, V.A., Eliott, J.A. and Hiller, J.E. (2014) Clinician Acceptance Is the Key Factor for Sustainable Telehealth Services. Qualitative Health Research, 5, 682-694. https://doi.org/10.1177/1049732314528809

Scientific Research Publishing

Submit or recommend next manuscript to SCIRP and we will provide best service for you:

Accepting pre-submission inquiries through Email, Facebook, LinkedIn, Twitter, etc. A wide selection of journals (inclusive of 9 subjects, more than 200 journals)

Providing 24-hour high-quality service

User-friendly online submission system

Fair and swift peer-review system

Efficient typesetting and proofreading procedure

Display of the result of downloads and visits, as well as the number of cited articles

Maximum dissemination of your research work

Submit your manuscript at: http://papersubmission.scirp.org/

Or contact ojn@scirp.org 